REVISTA

EPUEACIÓN
Revista Educación

ISSN: 0379-7082

ISSN: 2215-2644

revedu@gmail.com

Universidad de Costa Rica

Costa Rica

\title{
Creencias y actitudes del alumnado de secundaria obligatoria de la zona norte de Madrid sobre las causas de obesidad infantil
}

León-Sánchez, Rigoberto; Jiménez-Cruz, Blanca Elizabeth

Creencias y actitudes del alumnado de secundaria obligatoria de la zona norte de Madrid sobre las causas de obesidad infantil

Revista Educación, vol. 43, núm. 1, 2019

Universidad de Costa Rica, Costa Rica

Disponible en: http://www.redalyc.org/articulo.oa?id=44057415015

DOI: https://doi.org/10.15517/revedu.v43i1.28009

Esta obra está bajo una Licencia Creative Commons Atribución-NoComercial-SinDerivar 3.0 Internacional. 


\title{
Creencias y actitudes del alumnado de secundaria obligatoria de la zona norte de Madrid sobre las causas de obesidad infantil
}

\author{
High school Students' Beliefs and Attitudes on Childhood Obesity in Northern Madrid, Spain
}

Rigoberto León-Sánchez

Universidad Nacional Autónoma de México, México

rigobert@unam.mx

(iD http://orcid.org/0000-0002-0838-3507

Blanca Elizabeth Jiménez-Cruz

Universidad Nacional Autónoma de México, México

blancaelizabeth@gmail.com

(DD http://orcid.org/0000-0001-6947-4990
DOI: https://doi.org/10.15517/revedu.v43i1.28009

Redalyc: http://www.redalyc.org/articulo.oa?id=44057415015
Recepción: 21 Febrero 2017

Aprobación: 09 Diciembre 2018

\section{Resumen:}

En el presente trabajo se examinaron las creencias y actitudes de estudiantes de la Escuela Secundaria Obligatoria (ESO) procedentes de los cuatro grados escolares $(\mathrm{N}=348)$ sobre las causas de la obesidad, así como las actitudes que guardan hacia esta. Para recabar las creencias y actitudes de los participantes se utilizaron dos instrumentos diseñados ex profeso. Los resultados obtenidos con el cuestionario Creencias sobre las Causas de la Obesidad indican que los participantes tienden a ponderar factores como la voluntad por sobre los genéticos, ejercicio, etc., $\mathrm{F}(4.689,1627.115)=108.691, \mathrm{p}<.001$. Dicho resultado, asimismo, indica que para los participantes las personas que padecen obesidad son responsables de su condición, $\mathrm{t}(347)=-6.936, \mathrm{p}<.001$, d de Cohen $=0.54$. Por otra parte, los resultados obtenidos en el cuestionario Actitudes hacia la Obesidad Infantil mostraron que los participantes tienden a estar en desacuerdo $(84,84 \%)$ en atribuirles características negativas a las personas menores que padecen obesidad y solo un $35.25 \%$ está de acuerdo en atribuirles características positivas. Por último, se examina el papel de la educación formal en las creencias que sostienen los participantes acerca de las causas y las actitudes hacia la obesidad.

Palabras ClaVe: Creencias, Actitudes, Obesidad, Alumnos, Estigmatización.

\section{Abstract:}

This article examines beliefs and attitudes of students attending Compulsory High School Education (ESO, for its acronym in Spanish) in Spain on causes and their attitudes towards obesity. The students involved in the study represent four grades $(\mathrm{N}=$ 348). In order to gather student thoughts and attitudes, we designed two ex profeso questionnaires. The results collcted in one of the questionnaires, Beliefs About the Causes of Obesity, participants stated that they weighted factors such as willingness over genetics, exercise, etc, $\mathrm{F}(4,689,1627.115)=108,691, \mathrm{p}<.001$. Based on the results obtained, participants also believed that obese individually are ultimately responsible for their condition, $\mathrm{t}(347)=6936, \mathrm{p}<.001$. Moreover, the results of the questionnaire, Attitudes towards Childhood Obesity, reflect that $84.84 \%$ of participants tend to disagree that they used negative attributes when referring to obese children and only $35.25 \%$ agree that they referred to obese children using positive attributes. Finally, the role of formal education is discussed in helping change beliefs and improve student attitudes towards obesity.

KEYWORDS: Beliefs, attitudes, obesity, students, stigmatization.

\section{INTRODUCCIÓN}

El estudio del tema de la obesidad implica un abordaje desde distintos ángulos, siendo las creencias acerca de esta un elemento crucial cuando se trata de emprender su prevención y tratamiento.

Desde principios del siglo XXI la Organización Mundial de la Salud (WHO, 2000) llamó la atención sobre el incremento en la prevalencia del sobrepeso y la obesidad a nivel mundial, tanto en personas adultas como 
en personas menores ( $\mathrm{Ng}$ et ál., 2014), independientemente del nivel de desarrollo económico y tecnológico de los países (Ahrens et ál., 2014; Rivera et ál., 2013; Sánchez et ál., 2013; Vandevijvere et ál., 2015).

La obesidad constituye un problema de salud para el individuo tanto a nivel físico como psicológico, en tanto que aumenta significativamente el riesgo de padecer diversas enfermedades, como diabetes, ciertos tipos de cáncer, apnea del sueño, osteoartritis, problemas renales y ginecológicos, entre otros, al tiempo que supone una fuente de estrés frecuentemente asociada a la estigmatización y discriminación por el peso (Hans y Klein, 2017; Williams, Mesidor, Winters, Dubbert y Wyatt, 2015; Pont, Puhl, Cook y Slusser, 2017; Puhl y Suh, 2015a).

La 53ava Asamblea de la OMS del año 2000 planteó la necesidad de diseñar estrategias a nivel global que incidan en la dieta, la actividad física y la salud de las personas. Muchas de esas estrategias se han centrado en la población infantil y particularmente en el papel que tiene la escuela en la educación o reeducación de actitudes, conocimientos y conductas dirigidas hacia el cuidado y mantenimiento de la salud en general (WHO, 2002), y específicamente en aspectos relativos a la salud alimentaria (WHO, 2004).

La importancia que cobra la escuela en este proceso encaminado a prevenir el sobrepeso y la obesidad ha sido explicitada en diversas investigaciones (Centers for Disease Control, 2013; Clarke, Fletcher, Lancashire, Pallan y Adab, 2013; Sobol, Rabinowitz y Gross, 2013). De acuerdo con Fox (2010), dado que ninguna otra institución tiene un contacto tan continuo e intenso con un gran número de menores, la escuela se encuentra en una posición única para influir en la adquisición de patrones de vida saludable por parte de los educandos. No menos importante es el hecho de que el contexto escolar posibilita que se puedan explorar las actitudes, creencias, valores y conductas hacia la alimentación saludable y la actividad física que sostiene el estudiantado de educación básica (WHO, 2008).

El estudio de las creencias sobre las causas de la obesidad y sus actitudes hacia esta ha evidenciado que con mucha frecuencia los adultos legos (Casazza et ál., 2015; McFerran y Mukhopadhyay, 2013; Pearl y Lebowitz, 2014) y profesionales de la salud (Phelan et ál., 2015), así como los niños y adolescentes (Babboram, Mullan y Sharpe, 2011; Gonçalves et ál., 2012; Timotijevic et ál., 2018), tienden a considerar la obesidad como un rasgo corporal controlable a voluntad por el individuo, dejando de lado factores como la genética, las alteraciones metabólicas, la disponibilidad de lugares adecuados para realizar ejercicio, el acceso a alimentos saludables, la educación recibida, etcétera. Entre los aspectos que estas poblaciones consideran controlables por los individuos se encuentran la ingesta excesiva de comida y la falta de actividad física. En la mayoría de los estudios se destaca que la culpabilización a la persona obesa ocurre con independencia del peso corporal de quien emite el juicio (Casazza et ál., 2015; Puhl et ál., 2014); sin embargo, un estudio que mostró diferencia entre individuos normopeso e individuos con sobrepeso u obesidad, fue el de Gonçalves et ál. (2012). En este estudio se encontró que menores y adolescentes normopeso tendían a atribuir con mayor frecuencia causas conductuales a la obesidad, mientras que quienes tenían sobrepeso y obesidad creían con mayor frecuencia que se debía a factores genéticos.

La atribución de la obesidad a factores internos conduce directamente a la culpabilización del individuo obeso, generando no solo actitudes negativas y de menor empatía hacia él sino también conductas de discriminación (Phelan et ál., 2013; Puhl, Luedicke y Grilo, 2014; Puhl y Suh, 2015b).

En el caso particular de los niños se ha constatado que con frecuencia enfrentan ofensas verbales por parte de sus pares (e.g. apodos, comentarios despectivos y burlas), ofensas físicas (e.g. golpes, patadas y empujones) y exclusión social (e.g. ser ignorados o evitados, excluidos de actividades grupales, etc.), siendo más frecuente entre mujeres que entre hombres, y mediante el uso de expresiones más explícitas cuanto más temprana es la edad (Di Pasquale y Celsi, 2017). La victimización por estigmatización de la obesidad repercute a cualquier edad del individuo (Puhl y Suh, 2015a) pero, particularmente en la niñez y en la adolescencia, esta contribuye al aislamiento social, a la disminución de realización de actividad física, aumenta las probabilidades de comer compulsivamente, y por lo tanto, favorece el aumento de peso (Pont et al., 2017). 
Estudios como el de Khan, Tarrant, Weston, Shah y Farrow (2018), en el cual se probó con adultos el cambio en sus actitudes hacia la obesidad en función de las causas de esta, han mostrado que cuando se percibe que la persona obesa es culpable de su condición, la estigmatización prevalece; no obstante, una disminución en las actitudes negativas se presenta cuando la obesidad se achaca a cuestiones menos controlables por el individuo. En el estudio experimental realizado por Khan et ál., la mayor disminución en el grado de estigmatización de la obesidad se logró en quienes habían sido expuestos a información sobre las causas genéticas de la obesidad, seguidos de quienes se habían expuesto a información sobre las causas psicológicas y, por último, por quienes fueron expuestos a información sobre las causas conductuales de la obesidad, puesto que la conducta es percibida como un aspecto controlable por el propio individuo. De manera similar, Tiggemann y Anesbury (2000) encontraron que menores entre los 8 y los 12 años de edad tendían a asumir que la obesidad es controlable por los individuos y, consecuentemente, mientras más fuerte era esta asunción más fuertemente mantenían los estereotipos negativos hacia los individuos con sobrepeso u obesidad. Así, las creencias acerca de las causas de la obesidad parecen jugar un rol importante no solo en cuanto a los programas preventivos y de intervención para el tratamiento de la obesidad, sino para la reducción de su estigmatización.

Dado todo lo anteriormente expuesto, el objetivo del presente trabajo fue determinar las creencias que sostiene el alumnado de secundaria obligatoria de tres institutos de la zona norte de la comunidad de Madrid, sobre las causas de la obesidad infantil, así como el grado en el que se responsabiliza a los individuos que la padecen.

\section{MÉtodo}

Participantes. Se seleccionaron, por conveniencia, 348 estudiantes de la Escuela Secundaria Obligatoria (ESO) quienes cursaban los grados de primero $(\mathrm{n}=83)$, con un intervalo de edad de 12 a 13 años $(\mathrm{M}=$ $12.60, \mathrm{DE}=.679)$; segundo $(\mathrm{n}=89)$, con un intervalo de edad de 13 a 14 años $(\mathrm{M}=13.72, \mathrm{DE}=.848)$; tercero $(\mathrm{n}=87)$, con un intervalo de edad de 14 a 15 años $(\mathrm{M}=14.66, \mathrm{DE}=.717)$ y cuarto $(\mathrm{n}=89)$, con un intervalo de edad de 15 a 17 años $(\mathrm{M}=15.55$, $\mathrm{DE}=.723)$. Por sexo, la muestra estuvo compuesta por 174 mujeres y 174 hombres).

\section{Instrumentos}

a) Creencias sobre las causas de la obesidad. Se diseñó un cuestionario compuesto por 18 ítems con el objetivo de evaluar las creencias sobre las causas de la obesidad. Los ítems incluidos en el cuestionario fueron construidos a partir de la revisión de la literatura examinada (Brown, Stride, Psarou, Brewins y Thompson, 2007; Hilbert, Rief y Braehler, 2007; Klaczynski, Goold y Mudry, 2004; NeumarkSztainer, Story y Harris, 1999; Ogden y Flanagan, 2008). Los 18 ítems fueron organizados, ex profeso, en seis categorías diferentes (tres ítems en cada una; ver Tabla 1). Cada una de esas categorías representaba alguno de los factores que de acuerdo con la literatura (Brown et al., 2007; Hardus, van Vuuren, Crawford y Worsley, 2003; Sikorski et al., 2011) se han aducido como causas de la obesidad. 


\section{Tabla 1: Ejemplos de ítems por cada una de las categorías que conforman el cuestionario Creencias sobre las causas de la obesidad*.}

\begin{tabular}{|c|c|c|}
\hline & $\begin{array}{l}\text { Categorías } \\
\text { Causales }\end{array}$ & İtem \\
\hline 1 & Genética & $\begin{array}{l}\text { Muchas niñas* son obesas porque sus padres también } \\
\text { son obesos. }\end{array}$ \\
\hline 2 & Ejercicio & $\begin{array}{l}\text { Parece que a muchos niños* obesos no les gusta hacer } \\
\text { ejercicio. }\end{array}$ \\
\hline 3 & Comida & $\begin{array}{l}\text { Las niñas se vuelven obesas porque comen demasiada } \\
\text { comida grasosa. }\end{array}$ \\
\hline 4 & Personalidad & $\begin{array}{l}\text { Una razón importante para volverse obeso es ser } \\
\text { perezoso. }\end{array}$ \\
\hline 5 & Educación & $\begin{array}{l}\text { Algunos niños son obesos porque sus padres } \\
\text { acostumbran darles comida con mucha azúcar o grasa. }\end{array}$ \\
\hline 6 & Voluntad & $\begin{array}{l}\text { Casi todas las niñas obesas podrían bajar de peso si } \\
\text { realmente lo quisieran. }\end{array}$ \\
\hline
\end{tabular}

*Los ítems fueron redactados utilizando el término niña en el caso de los cuestionarios aplicados a las mujeres y con el término niño en el caso de los varones. Esto se decidió, principalmente, con el fin de evitar posibles sesgos en las respuestas.

Fuente: Elaboración propia

Cada ítem fue calificado por los participantes en una escala Likert de cuatro puntos: (1) Totalmente en desacuerdo; (2) En desacuerdo; (3) De acuerdo; (4) Totalmente de acuerdo. Por ende, la amplitud de los puntajes se distribuyó, en cada una de las categorías, de un mínimo de 3 a un máximo de 12 puntos. A mayor puntaje, mayor acuerdo con la categoría causal.

a) Actitudes hacia la obesidad infantil. Tomando como modelo el cuestionario de Neumark, Story y Harris (1999), se construyeron 16 ítems con el fin de evaluar las actitudes hacia los niños obesos. Sean actitudes positivas, por ejemplo: Las niñas obesas tienen tantas amigas como las niñas delgadas o negativas, por ejemplo: Los niños obesos tienden a ser muy problemáticos en sus relaciones con otros niños. Los ítems fueron redactados, como en el caso del instrumento anterior, utilizando el término niña en el caso de los cuestionarios aplicados a las mujeres y el término niño en el caso de los varones. Los ítems fueron respondidos por los participantes en una escala Likert de cuatro puntos: (1) Totalmente en desacuerdo; (2) En desacuerdo; (3) De acuerdo, y (4) Totalmente de acuerdo. Los reactivos cuya redacción indicaba actitudes positivas, se calificaron inversamente, es decir, (4) Totalmente en desacuerdo; (3) En desacuerdo; (2) De acuerdo, y (1) Totalmente de acuerdo. Para el análisis de los reactivos, primero se realizó un análisis del índice de fiabilidad de los 16 ítems. 
Dicho análisis, mediante el Alpha de Cronbach, indicó un $\alpha=.662$. Se eliminaron cuatro ítems cuya relación ítem-total era menor a .20. Un nuevo análisis indicó un $\alpha=.740$ para 12 ítems oscilando entre .305 y .505 . Posteriormente, con el fin de evaluar la estructura factorial del cuestionario se realizó un Análisis Factorial Exploratorio (EFA) utilizando como método de extracción de factores un Análisis de Componentes Principales (ACP). Al evaluar la pertinencia de los datos a este análisis se obtuvo un estadístico de adecuación muestral de Kaiser-Mayer-Olkin (KMO) de .801 y el test de esfericidad de Bartlett resultó estadísticamente significativo: $\chi 2=609.029 ; \mathrm{gl}=66 ; \mathrm{p}<.001$. Dicho resultado mostró la pertinencia de realizar un análisis factorial. Mediante el Análisis de Componentes Principales y una rotación Varimax se extrajeron tres factores que explicaban el 48.04\% de la varianza. Para efectos de determinar empíricamente la cantidad de factores se consideró el criterio de KaiserGuttman, es decir, se consideraron solo aquellos factores que presentaron auto-valores $>1,0$. Solo uno de los ítems (16) saturó en dos factores decidiéndose dejarlo en el factor 2 dado que en este saturó más alto y tenía mayor coherencia conceptual. No obstante, y dado que en el factor 2 uno de los ítems (La mayoría de los niños obesos son tan buenos estudiantes como los niños delgados) no era conceptualmente coherente con los otros contenidos en el factor se decidió eliminarlo y volver a correr el análisis. En este nuevo análisis se obtuvo un estadístico de adecuación muestral de KaiserMayer-Olkin (KMO) de .789. El test de esfericidad de Bartlett resultó estadísticamente significativo: $\chi^{2}=526.690 ; \mathrm{gl}=55, \mathrm{p}<.001$ y las comunalidades fueron mayores a .40 . Mediante el Análisis de Componentes Principales y una rotación Varimax se extrajeron 3 factores que explicaron el $49.50 \%$ de la varianza total (Tabla 2). El Factor 1, compuesto por cuatro ítems, se refiere a la exclusión de los niños obesos de las Relaciones sociales (RELAS) con sus pares. El Factor 2, compuesto por cuatro ítems, representa la atribución de Características Negativas (CNEG) hacia los niños obesos, mientras que el Factor 3, compuesto por tres ítems, se refiere a la atribución de Características Positivas (CPOS). 
Tabla 2: Estructura factorial del Cuestionario actitudes hacia la obesidad.

\begin{tabular}{|c|c|c|c|c|}
\hline \multirow{2}{*}{ Ítem } & & \multicolumn{3}{|c|}{ Factores } \\
\hline & & $\mathrm{F} 1$ & $\mathrm{~F} 2$ & F3 \\
\hline 08 & 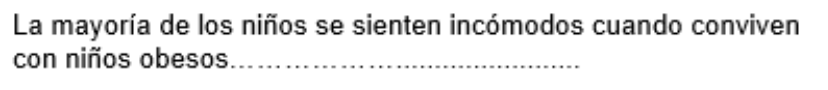 & .739 & & \\
\hline 05 & $\begin{array}{l}\text { La mayoría de los niños delgados no quisieran tener como amigo } \\
\text { a un niño obeso. }\end{array}$ & .620 & & \\
\hline 15 & $\begin{array}{l}\text { Los niños obesos tienden a ser muy problemáticos en sus } \\
\text { relaciones con otros niños................................ }\end{array}$ & .602 & & \\
\hline 16 & 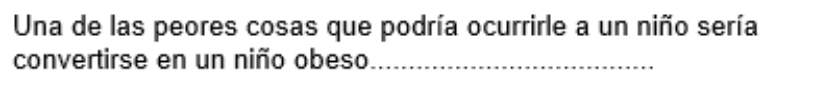 & .586 & & \\
\hline 06 & La mayoría de los niños obesos son desaseados............ & & .669 & \\
\hline 11 & Los niños obesos son personas irresponsables ............. & & 602 & \\
\hline 03 & 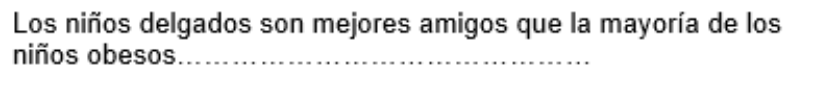 & & .566 & \\
\hline 04 & $\begin{array}{l}\text { Los niños obesos, como alumnos, no pueden ser tan exitosos en } \\
\text { la escuela como los niños delgados............ }\end{array}$ & & .524 & \\
\hline 01 & 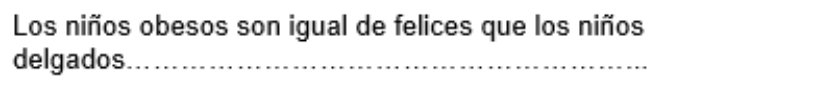 & & & .721 \\
\hline 07 & $\begin{array}{l}\text { Verdaderamente, los niños obesos son tan sinceros como los } \\
\text { niños delgados............................................... }\end{array}$ & & & .673 \\
\hline 10 & 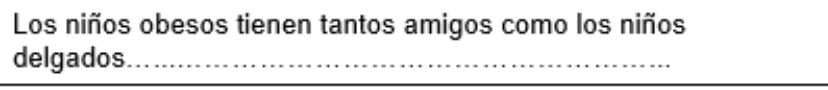 & & & .643 \\
\hline \multicolumn{2}{|c|}{ Valor propio } & 2.98 & 1.24 & 1.21 \\
\hline \multicolumn{2}{|c|}{ Porcentaje de la varianza explicada } & 18.60 & 16.31 & 14.58 \\
\hline \multicolumn{2}{|c|}{ Alpha de Cronbach } & .608 & .620 & .576 \\
\hline
\end{tabular}

Fuente: Elaboración propia

Procedimiento. Se contactó con los directores de tres centros de Educación Secundaria Obligatoria (ESO) de la zona norte de Madrid. En una reunión de profesores, la dirección de cada centro les explicó el propósito del estudio y les pidió su ayuda para la aplicación de los cuestionarios. El profesorado acordó informar a sus estudiantes de los objetivos del estudio y obtener su consentimiento para responder los cuestionarios. Únicamente se les dio el cuestionario a aquellos estudiantes que habían consentido contestarlo. La aplicación se realizó en el salón de tutorías del Centro y fue respondido de forma colectiva por quienes asistían en ese momento. El salón de tutorías, el cual era utilizado cuatro veces por semana (dos sesiones en la mañana y dos sesiones en la tarde), tenía una capacidad para 30 alumno. La recolección de los cuestionarios se realizó a lo largo de la última semana de septiembre de 2015. La aplicación de los mismos se llevó a cabo entre 15 y 20 minutos. 
Los tres centros pertenecen a la misma zona escolar y se encuentran muy cercanos unos de otros, de hecho, comparten docentes y muchas de las actividades académicas extracurriculares son planeadas en conjunto con la participación de los docentes y de los alumnos. Dado ese hecho, no se diferenció entre estudiantes de uno u otro Centro. Únicamente se tomaron en cuenta las variables edad, gado escolar y sexo con el fin de examinar su impacto en las respuestas.

\section{Resultados}

Causas de la obesidad. Con la ayuda del paquete SPSS-20 se llevaron a cabo los diferentes análisis estadísticos. Primero, se realizó un ANOVA de un factor con medidas repetidas para indagar si existían diferencias entre las medias de las seis categorías. No se encontró esfericidad $(\mathrm{W}=.853, \mathrm{p}<.001)$ por lo que se reporta el criterio de Greenhouse-Geisser, $\mathrm{F}(4.689,1627.115)=108.691, \mathrm{p}<.001$ (potencia observada = 1; $\eta 2$ parcial $=.239$ ). Un ajuste de los niveles críticos mediante la corrección de Bonferroni (véase Tabla 3 ) mostró que las diferencias se dieron en todos los pares comparados ( $\mathrm{p}$-valor $<.05$ ), excepto en los pares Ejercicio-Comida y Personalidad-Educación. 
Tabla 3: Comparaciones múltiples de las seis categorías (con el ajuste de Bonferroni) que componen el cuestionario Creencias sobre las causas de la obesidad.

\begin{tabular}{|c|c|c|c|c|c|c|}
\hline \multirow[b]{2}{*}{ (I) Categorías } & \multirow[b]{2}{*}{ (J) Categorías } & \multirow{2}{*}{$\begin{array}{l}\text { Diferencia } \\
\text { medias (I-J) }\end{array}$} & \multirow{2}{*}{$\begin{array}{l}\text { de Error } \\
\text { típico }\end{array}$} & \multirow[b]{2}{*}{ Sig. } & \multicolumn{2}{|c|}{ IC $95 \%$} \\
\hline & & & & & $\mathrm{LI}$ & LS \\
\hline \multirow[t]{5}{*}{$1 \mathrm{GEN}$} & 2 EJE & $-.727^{*}$ & .124 & .000 & -1.095 & -.359 \\
\hline & $3 \mathrm{COM}$ & $-.563^{*}$ & .129 & .000 & -.945 & -.181 \\
\hline & 4 PER & $-1.431^{\circ}$ & .131 & .000 & -1.817 & -1.045 \\
\hline & 5 EDU & $-1.454^{\circ}$ & .117 & .000 & -1.801 & -1.107 \\
\hline & $6 \mathrm{VOL}$ & $-2.534^{*}$ & .140 & .000 & -2.948 & -2.121 \\
\hline \multirow[t]{4}{*}{2 EJE } & $3 \mathrm{COM}$ & .164 & .103 & 1.000 & -.140 & .467 \\
\hline & 4 PER & $-.704^{*}$ & .109 & .000 & -1.026 & -.382 \\
\hline & 5 EDU & $-.727^{*}$ & .111 & .000 & -1.056 & -.398 \\
\hline & $6 \mathrm{VOL}$ & $-1.807^{*}$ & .116 & .000 & -2.150 & -1.465 \\
\hline \multirow[t]{3}{*}{$3 \mathrm{COM}$} & 4 PER & $-.868^{*}$ & .109 & .000 & -1.189 & -.547 \\
\hline & 5 EDU & $-.891^{*}$ & .117 & .000 & -1.238 & -.544 \\
\hline & $6 \mathrm{VOL}$ & $-1.971^{*}$ & .124 & .000 & -2.338 & -1.604 \\
\hline \multirow[t]{2}{*}{4 PER } & 5 EDU & -.023 & .119 & 1.000 & -.376 & .330 \\
\hline & $6 \mathrm{VOL}$ & $-1.103^{*}$ & .125 & .000 & -1.474 & -.733 \\
\hline $5 \mathrm{EDU}$ & $6 \mathrm{VOL}$ & $-1.080^{\circ}$ & .123 & .000 & -1.443 & -.718 \\
\hline
\end{tabular}

Nota: Sig. = Ajuste de Bonferroni para comparaciones múltiples; IC = Intervalo de Confianza; $\mathrm{LI}$ = Límite Inferior; LS = Límite Superior. Categorías causales: 1 GEN = Genética; 2 EJE = Ejercicio; 3 COM = Comida; 4 PER = Personalidad; 5 EDU = Educación; 6 VOL = Voluntad .

$$
{ }^{*} p<.05
$$

\section{Fuente: Elaboración propia}

Los datos obtenidos muestran que la Voluntad tiene una mayor incidencia en las causas de la obesidad $(\mathrm{M}=9.26, \mathrm{DE}=1.83)$ mientras que la Genética tiene una menor incidencia $(\mathrm{M}=6.73, \mathrm{DE}=1.73)$. Entre ambas categorías causales quedaron: Educación $(\mathrm{M}=8.18, \mathrm{DE}=1.57)$, Personalidad $(\mathrm{M}=8.16, \mathrm{DE}=1.82)$, Ejercicio $(\mathrm{M}=7.45, \mathrm{DE}=1.58)$ y Comida $(\mathrm{M}=7.29, \mathrm{DE}=1.70)$.

Asimismo, con el propósito de valorar las diferencias entre las respuestas de los participantes se efectuó un análisis multivariante de varianza (MANOVA) en el cual las variables dependientes fueron las seis categorías causales mientras que los factores fueron las variables sexo (2) y grado escolar (4). Debe mencionarse que un análisis de las variables edad y grado escolar mostró una fuerte correlación entre ellas $(\mathrm{r}=.819, \mathrm{p}=.001)$. Por tal razón se decidió examinar únicamente una de ellas con el fin de evitar redundancias en el análisis. Los resultados indicaron un efecto significativo de la variable sexo únicamente en las categorías causales Ejercicio 
$(\mathrm{F} 1,347=11.422, \mathrm{p}=.001)$ y Comida $(\mathrm{F} 1,347=10.798, \mathrm{p}=.001)$, siendo que en ambos casos las medias de los hombres $(\mathrm{M}=7.76, \mathrm{DE}=1.58 ; \mathrm{M}=7.58, \mathrm{DE}=1.80$, respectivamente $)$ fueron más altas que las de las mujeres $(\mathrm{M}=7.15, \mathrm{DE}=1.57 ; \mathrm{M}=7.01, \mathrm{DE}=1.54$, respectivamente). Es decir, los hombres ponderan más el ejercicio y la comida como causas de la obesidad que las mujeres. En las restantes categorías causales no se encontraron diferencias estadísticamente significativas ( $\mathrm{p}$-valor $>.05$ ). Por otro lado, se observó un efecto significativo de la variable grado escolar únicamente en la categoría causal Comida, $\mathrm{F}(3,348)=4.528$, $\mathrm{p}=.004)$. La prueba de Tukey indicó que las diferencias se dan únicamente entre primero y cuarto grados, $\mathrm{p}=.003$, IC 95\% [.2400, 1.5478]. Es decir, el grado escolar tuvo repercusión estadística en la valoración de las causas de la obesidad, siendo los participantes de primer grado $(\mathrm{M}=7.75, \mathrm{DE}=1.87)$ quienes tendieron a dar más importancia a la comida (cantidad y tipo) como causa de la obesidad que los de cuarto grado $(\mathrm{M}=$ 6.86, $\mathrm{DE}=1.74)$. Por otro lado, no se encontró un efecto de la interacción sexo*grado escolar ( $\mathrm{p}$-valor $>.05)$.

Posteriormente, los 18 ítems que componían cada una de las seis categorías causales se conjuntaron en dos dimensiones; a saber, aquellos que implican que los individuos son no-responsables de su condición, compuesto por 6 ítems (v.gr.: Generalmente, los niños obesos tienen enfermedades que los volvieron obesos; Muchos niños obesos aprendieron malos hábitos alimentarios de sus padres). $\mathrm{O}$, por el contrario, aquellos que implican que son responsables, compuesto por 12 ítems (v. gr.: Los niños que son obesos lo son porque comen más que los niños delgados; Una razón importante para volverse obeso es ser perezoso). Dado que el número de ítems que componen cada una de las dimensiones era distinto se promediaron los ítems con el fin de poder comparar las medias. Una prueba t para muestras relacionadas encontró diferencias estadísticamente significativas entre las medias de Responsable $(\mathrm{M}=2.68, \mathrm{DE}=.383)$ y No-Responsable $(\mathrm{M}=2.48, \mathrm{DE}$ $=.348), \mathrm{t}(347)=-6.936, \mathrm{p}<.001, \mathrm{~d}$ de Cohen $=0.54$; en otras palabras, los participantes tendieron a considerar que los individuos obesos son responsables de su condición.

Asimismo, con el propósito de valorar las diferencias entre las respuestas de los participantes se efectuó un análisis multivariante de varianza (MANOVA) en el cual las variables dependientes fueron las dimensiones (2) Responsable y No-Responsable mientras que los factores fueron las variables sexo (2) y grado escolar (4). Los resultados indicaron un efecto significativo de la variable sexo únicamente en la dimensión Responsable, $\mathrm{F}(1,347)=7.299, \mathrm{p}=.007)$, siendo que la media de los hombres $(\mathrm{M}=2.74, \mathrm{DE}=.396)$ fue más alta que las de las mujeres $(\mathrm{M}=2.62, \mathrm{DE}=.361)$. Es decir, los hombres tienden a responsabilizar más a las personas obesas que las mujeres. Por otro lado, se observó un efecto significativo de la variable grado escolar también en la dimensión Responsable, $\mathrm{F}(3,348)=3.445, \mathrm{p}=.017$. La prueba de Tukey indicó que las diferencias se dan únicamente entre primero y cuarto grados, $\mathrm{p}=.010$, IC 95\% [.030, .336]. Es decir, el grado escolar tuvo repercusión estadística en la valoración de las causas de la obesidad, siendo los participantes de primer grado $(\mathrm{M}=2.78, \mathrm{DE}=.377)$ quienes tienden a responsabilizar más a las personas obesas que los de cuarto grado $(\mathrm{M}=2.60, \mathrm{DE}=.425)$. No se encontró un efecto de la interacción sexo*grado escolar ( $\mathrm{p}$-valor $>.05)$.

\section{Actitudes hacia la obesidad infantil.}

Se realizó un ANOVA de un factor con medidas repetidas para indagar si existían diferencias entre las medias de los tres factores: Relaciones sociales (RELAS), Características negativas (CNEG) y Características positivas (CPOS). No se encontró esfericidad $(\mathrm{W}=.965, \mathrm{p}<.002)$ por lo que se reporta el criterio de Greenhouse-Geisser, $\mathrm{F}(1.933,670.700)=101.605, \mathrm{p}<.001$ (potencia observada $=1 ; \eta 2$ parcial $=.226$ ). Un ajuste de los niveles críticos mediante la corrección de Bonferroni encontró diferencias estadísticamente significativas ( $\mathrm{p}$-valor $<.05)$ entre $\mathrm{F} 1(\mathrm{M}=2.15, \mathrm{DE}=.622)$ y F2 $(\mathrm{M}=1.67, \mathrm{DE}=.522)$ y entre F2 y F3 $(\mathrm{M}=2.14, \mathrm{DE}=.706)$ pero no entre $\mathrm{F} 1 \mathrm{y} \mathrm{F} 3$.

Posteriormente, se recodificaron los puntajes de la escala Likert con el fin de reorganizar las respuestas. Así, los valores 1 y 2 fueron recodificados como 1 (Desacuerdos) mientras que 3 y 4 fueron recodificados como 2 (Acuerdos). Un análisis de las respuestas mostró que el $66.31 \%$ de los participantes muestran su 
desacuerdo con los enunciados que componen el Factor 1 (RELAS); es decir, están en contra de que se excluya socialmente a los niños que padecen obesidad. Sin embargo, en el ítem 16 (Una de las peores cosas que podría ocurrirle a un niño sería convertirse en un niño obeso) el $51.7 \%$ de los participantes dijo estar de acuerdo con ese enunciado. Una prueba de Kruskal-Wallis mostró la existencia de diferencias estadísticamente significativas entre los rangos promedio de los cuatro grados escolares, $\chi 2(3,348)=12.603, p<.05$. Siendo los estudiantes de primero (185.13) y segundo (193.98) grados quienes están más de acuerdo que los de tercero (168.50) y cuarto (150.97) con ese enunciado. Por su parte, en el Factor 2 (CNEG), el 84.84\% dijo estar en desacuerdo con los enunciados. A saber, la mayoría de los participantes no les atribuye características negativas a los niños que padecen obesidad. No obstante, en el Factor 3 (CPOS), solo el 35.25\% de los participantes está de acuerdo en atribuirles características positivas. De hecho, únicamente el 17.2\% dice estar de acuerdo con el enunciado: Verdaderamente, los niños obesos son tan sinceros como los niños delgados.

Por último, y dado que se había preguntado a los participantes por los días a la semana que hacían ejercicio, a partir de una distribución intercuartilar se conformaron tres grupos con el 64.1\% de los participantes (223): G1EJER, quienes lo hacían dos días a la semana, G2EJER, quienes lo hacían cuatro y G3EJER, quienes lo hacían seis. Una prueba ANOVA de un factor indicó que únicamente existen diferencias estadísticamente significativas entre las medias de los tres grupos en la categoría causal Comida, $\mathrm{F}(2,222)=3.328, \mathrm{p}=.038$. La prueba de Tukey indicó que las diferencias se encuentran entre los alumnos de G1EJER y G3EJER, $p$ $=.037, \mathrm{IC} 95 \%$ [- 1.3636, -.0342]. Es decir, quienes hacen más ejercicio (G31EJER) son quienes consideran, en mayor medida, que la comida (cantidad y tipo) es un aspecto muy relevante en las causas de la obesidad y, por ende, que el control sobre la conducta, es decir, hacer más ejercicio impacta en el control del peso.

\section{Discusión y CONCLUSIONES.}

La evidencia existente en la literatura muestra que para las personas la ganancia de peso es controlable por los individuos y, en consecuencia, asumen que si los individuos con sobrepeso/obesidad no tienen la motivación o la voluntad suficientes para bajar de peso entonces son responsables de su condición (Casazza et ál., 2015). Por ello, quizá no es sorprendente que las actitudes hacia este grupo de personas se encuentren influenciadas por el grado en que la obesidad es percibida como controlable por el individuo, de tal manera que si las personas creen que la obesidad se encuentra fuera del control del individuo tienden a tener actitudes más positivas hacia estas; no obstante, esto cambia si se asume que la obesidad cae bajo el control del individuo (Puhl et al., 2014; Puhl y Suh, 2015b). Es interesante hacer la observación de que este tipo de atribuciones se da con independencia de si son niños o adultos quienes las hacen (Khan et al., 2018; Tiggemann y Anesbury, 2000).

Los resultados que hemos obtenido en el presente estudio coinciden con los aportados por la literatura. De las seis categorías causales propuestas a los participantes, estos se decantaron por elegir la categoría Voluntad como aquella que mayormente incide en la obesidad. Y si bien la categoría Educación ocupó el segundo lugar en la elección de factores causales, es interesante notar que es la categoría Personalidad la que ocupa el tercer sitio. Interesante, porque las categorías causales Voluntad y Personalidad apuntan, precisamente, a responsabilizar al individuo obeso de su condición. Así, por ejemplo, en dos de los ítems incluidos en la categoría causal Voluntad (Si las niñas obesas tuvieran más fuerza de voluntad, serían capaces de comer menos y Los niños obesos no son capaces de controlar la cantidad de comida que comen), los participantes dijeron estar de acuerdo; el $83.9 \%$ de las veces en el primer caso y $72.7 \%$ en el segundo. De la misma manera, en un ítem perteneciente a la categoría causal Personalidad (Una razón importante para volverse obeso es ser perezoso), el 60.4\% de los participantes dijo estar de acuerdo. Esta tendencia se reafirma con el hecho de que los participantes, tienden a considerar la Dimensión Responsable por sobre la Dimensión No-Responsable. En suma, los participantes del estudio ponderan en mayor medida la carencia de fuerza de voluntad y la falta de motivación como causas de la obesidad, mientras que dejan en los últimos lugares categorías causales como 
la Genética. Por otra parte, debe mencionarse que son los participantes de primer grado quienes atribuyen una mayor responsabilidad a la persona obesa que los de cuarto grado.

Esta inclinación en la asignación de la causalidad quizás también explique por qué los participantes muestran no solo una mayor tendencia a atribuir a los individuos la responsabilidad de su condición sino también una mayor renuencia a atribuirles características positivas. Que solo el 35.25\% de los participantes les atribuya propiedades positivas a las personas obesas atestigua ese hecho. De manera similar, es llamativo que la mitad de los participantes esté de acuerdo con el enunciado: Una de las peores cosas que podría ocurrirle a un niño sería convertirse en un niño obeso. Más llamativo porque ese resultado se suma a aquel que muestra que solo el $35 \%$ le otorga característica positivas y que solo el $17 \%$ está de acuerdo en que los niños obesos son igual de sinceros que los niños delgados. En todos estos casos, son los participantes de primero y segundo grados quienes muestran más su acuerdo que los participantes de tercero y cuarto grados. En resumen, la asignación causal centrada en la responsabilidad del individuo no se encuentra separada de la atribución negativa y, en última instancia, de la subsiguiente estigmatización.

En concordancia, participantes que reportaron hacer más ejercicio fueron quienes consideraron en mayor medida que la comida (cantidad y tipo) es un factor relevante en la determinación de la obesidad. Trabajos como los de McFerran y Mukhopadhyay (2013) que han examinado las teorías implícitas sobre la ganancia/ pérdida de peso han encontrado esta misma relación, a saber, el ejercicio impacta en el control de peso equilibrando la cantidad de energía que se ingiere. Pero, en nuestro caso, también deja entrever que para los participantes del estudio hacer ejercicio se relaciona muy estrechamente con el dominio sobre la conducta individual y, por ende, es el individuo el responsable de controlar su peso. Así, si bien atribuirle la responsabilidad de su condición a la persona obesa y no reconocerle características positivas es una tendencia general de los participantes de este estudio (independiente del sexo, del grado escolar e incluso de los hábitos, por ejemplo, hacer ejercicio), es interesante hacer notar que dicha tendencia parece aminorar conforme aumenta el nivel académico. Ello parece indicar que la educación juega un papel muy importante y que desde la escuela se podría ampliar el conocimiento del estudiantado acerca de las causas de la obesidad y, en consecuencia, lograr que estos consideren otros factores que inciden sobre las causas de la obesidad (como la genética), y que no conciban el problema de la obesidad como una relación causal directa sino como un problema multifactorial. Con ello también se incidiría, tomando como caso la obesidad y el proceso nutritivo, en el fortalecimiento del pensamiento científico de los estudiantes. Si esto es así, entonces el objetivo de la escuela sería diseñar e implementar programas que tiendan a aumentar el conocimiento sobre las causas de la obesidad y la generación de programas que tengan como meta eliminar las actitudes negativas que dispensan los estudiantes hacia las personas que padecen sobrepeso u obesidad y que conducen a la manifestación de actitudes y conductas no deseables como la estigmatización y el bullying. Tal empresa no es sencilla de llevar a cabo, pero por lo menos los datos obtenidos permiten vislumbrar cuáles son los conocimientos previos que el alumnado sostiene y que quizás, como pasa en otros campos, son un fuerte obstáculo para el aprendizaje de nuevos contenidos.

Por último, si bien los resultados obtenidos en el presente estudio son similares a los obtenidos en otras investigaciones que han examinado las creencias sobre las causas de la obesidad y las actitudes hacia las personas que la padecen, es pertinente mencionar que el estudio tiene una serie de limitaciones que deben tenerse presente al momento de interpretar los datos. Una de esas limitaciones se centra en la manera en la cual se aplicaron los cuestionarios. Dado que fueron los responsables de las tutorías los encargados de aplicar los cuestionarios y no los investigadores, ello imposibilitó que se pudieran escuchar los comentarios de los participantes. De suyo valioso porque esos comentarios, cuando se dan, recogen información adicional que es imposible recopilar a través de un cuestionario. La otra limitación está relacionada con la falta de un estudio previo que permitiera probar los instrumentos. Si bien un análisis factorial es una prueba pertinente, su uso no soluciona los déficits que puede tener un instrumento en su diseño. Ambas limitaciones deberán ser corregidas para futuras investigaciones. 


\section{REFERENCIAS}

Ahrens, W., Pigeot, I., Pohlabeln, H., De Henauw, S., Lissner, L., Molnár, D. y Siani, A. (2014). Prevalence of overweight and obesity in European children below the age of 10.International Journal of Obesity,38, S99-S107. doi: $10.1038 /$ ijo.2014.140

Brown, I., Stride, C., Psarou, A., Brewins, L. y Thompson, J. (2007). Management of obesity in primary care: nurses' practices, beliefs and attitudes. Journal of Advanced Nursing, 59(4), 329-341. doi: 10.1111/ j.1365-2648.2007.04297.x

Casazza, K., Brown, A., Astrup, A., Bertz, F., Baum, C., Bohan, M. y Allison, D. B. (2015). Weighing the evidence of common beliefs in obesity research. Critical Reviews in Food Science and Nutrition, 55, 2014-2053, doi: $10.1080 / 10408398.2014 .922044$

Centers for Disease Control (2013). Make a difference at your school. Chronic Disease. Paper 31. http:// digitalcommons.hsc.unt.edu/disease/31

Clarke, J. Fletcher, B., Lancashire, E., Pallan, M., y Adab, P. (2013). The views of stakeholders on the role of the primary school in preventing childhood obesity: A qualitative systematic review. Obesity Reviews, 14, 975-988. doi: doi:10.1111/obr.12058

Di Pasquale, R., y Celsi, L. (2017). Stigmatization of overweight and obese peers among children. Frontiers in Psychology, 8, doi: 10.3389/fpsyg.2017.00524

Fox, M. K. (2010). Improving food environments in schools: tracking progress. Journal of the American Dietetic Association, 110(7), 1010-1013.

Gonçalves, H., González, D. A., Araújo, C. P., Muniz, L., Tavares, P., Assunção, M. C. ...Hallal, P. C. (2012). Adolescents' perception of causes of obesity: Unhealthy lifestyles or heritage? Journal of Adolescent Health, 51, doi: 10.1016/j.jadohealth.2012.08.015

Hans-Rudolf, B., y Klein, S. (2017). Advances in obesity: Causes, consequences, and therapy. Gastroenterology, 152, 1635-1637. doi: 10.1053/j.gastro.2017.03.045

Hardus, P.M., van Vuuren, C.L., Crawford, D. y Worsley, A. (2003). Public perceptions of the causes and prevention of obesity among primary school children. International Journal of Obesity, 27, 1465-1571.

Hilbert, A., Rief, W. y Braehler, E. (2007). What determines public support of obesity prevention? Journal of Epidemiology y Community Health, 61, 585-590. doi: 10.1136/ jech.2006.050906

Khan, S. S., Tarrant, M., Weston, D., Shah, P., y Farrow, C. (2018). Can raising awareness about the psychological causes of obesity reduce obesity stigma? Health Communication, 33(5), 585-592. doi: 10.1080/10410236.2017.1283566

Klaczynski, P. A., Goold, K. W. y Mudry, J. J. (2004). Culture, Obesity Stereotypes, Self-esteem, and the "thin ideal": Social Identity Perspective. Journal of Youth and Adolescence, 33, 307-317.

McFerran, B. y Mukhopadhyay, A. (2013). Lay theories of obesity predict actual body mass. Psychological Science, 24(8), 1428-1436. doi: 10.1177/0956797612473121

Neumark-Sztainer, D., Story, M. y Harris, T. (1999). Beliefs and attitudes about obesity among teachers and school health care providers working with adolescents. Journal of Nutrition Education, 31(1), 3-9.

Ng, M., Fleming, T., Robinson, M., Thomson, B., Graetz, N., Margono, C. y.Gakidou, E. (2014). Global, regional, and national prevalence of overweight and obesity in children and adults during 1980-2013: A systematic analysis for the Global Burden of Disease Study 2013. The Lancet, 384, 766-781. doi: 10.1016/S0140-6736(14)60460-8

Ogden, J. y Flanagan, Z. (2008). Beliefs about the causes and solutions to obesity: A comparison of GPs and lay people. Patient Education and Counseling, 71(1), 72-78. doi: 10.1016/j.pec.2007.11.022

Pearl, R. L., y Lebowitz, M. S. (2014). Beyond personal responsibility: Effects of causal attributions for overweight and obesity on weight-related beliefs, stigma, and policy support. Psychology y Health, 29(10), 1176-1191. doi: $10.1080 / 08870446.2014 .916807$ 
Phelan, S. M., Burgess, D. J., Burke, S. E., Przedworski, J. M., Dovidio, J. F., Hardeman, R., (2015). Beliefs about the causes of obesity in a national sample of 4th year medical students. Patient Education and Counseling, 98, doi: 10.1016/j.pec.2015.06.017

Phelan, S. M., Dovidio, J. F., Puhl, R. M., Burgess, D. J., Nelson, D. B., Yeazel, M. W. ...van Ryn, M. (2013). Implicit and explicit weight bias in a national sample of 4,732 medical students: The medical student CHANGES study. Obesity, 22(4), 1201-1207. doi:10.1002/oby.20687

Pont, S. J., Puhl, R., Cook, S. R., y Slusser, W. (2017). Stigma experienced by children and adolescents with obesity. Pediatrics, 140(6). doi: 10.1542/peds.2017-3034

Puhl, R., Luedicke, J., y Grilo, C. M. (2014). Obesity bias in training: Attitudes, beliefs, and observations among advanced trainees in professional health disciplines. Obesity, 22(4), 1008-1015. doi: 10.1002/oby.20637

Puhl, R., y Suh, Y. (2015a). Health consequences of weight stigma: Implications for obesity prevention and treatment. Current Obesity Reports, 4, 182-190. doi: 10.1007/s13679-015-0153-z

Puhl, R., y Suh, Y. (2015b). Stigma and eating and weight disorders. Current Psychiatry Reports, 17(10), doi: 10.1007/ s11920-015-0552

Rivera, J. Á., de Cossío, T. G., Pedraza, L. S., Aburto, T. C., Sánchez, T. G., y Martorell, R. (2013). Childhood and adolescent overweight and obesity in Latin America: a systematic review. The Lancet Diabetes y Endocrinology, 2(4), 321-332. doi: 10.1016/S2213-8587 (13)70173-6

Sánchez-Cruz, J. J., Jiménez-Moleón, J. J., Fernández-Quesada, F., y Sánchez, M. J. (2013). Prevalence of Child and Youth Obesity in Spain in 2012. Revista Española de Cardiologia, 66(5), 371-376.

Sikorski, C., Luppa, M., Kaiser, M., Glaesmer, H., Schomerus, G., Köning. H-H. y Riedel-Heller, S. G. (2011). The stigma of obesity in the general public and its implications for public health. A systematic review. BMC Public Health, 11:661. doi:10.1186/1471-2458-11-661

Sobol-Goldberg, S., Rabinowitz, J., y Gross, R. (2013). School-based obesity prevention programs: A meta-analysis of randomized controlled trials. Obesity, 21(12), 2422-2428. doi: 10.1002/oby.20515

Tiggemann, M. y Anesbury, T. (2000). Negative stereotyping of obesity in children: The role of controllability beliefs. Journal of Applied Social Psychology, 30(9), 1977-1993. doi: 10.1111/j.1559-1816.2000.tb02477.x

Timotijevic, L., Acuna\#Rivera, M., Gemen, R., Kugelberg, S., McBarron, K., Raats, M. M., y Zolotonosa, M. (2018). Adolescents' Perspectives on Personal and Societal Responsibility for Childhood Obesity-The Study of Beliefs through 'Serious' Game (PlayDecide). Children y Society, 32, 405-416. doi: 10.1111/chso.12271

Vandevijvere, S., Chow, C. C., Hall, K. D., Umali, E., y Swinburn, B. A. (2015). Increased food energy supply as a major driver of the obesity epidemic: a global analysis. Bulletin of the World Health Organization, 93(7), 446-456. doi: 10.2471/BLT.14.150565

Williams, E. P., Mesidor, M., Winters, K., Dubbert, P. M., y Wyatt, S. B. (2015). Overweight and obesity: Prevalence, consequences, and causes of a growing public health problem. Current Obesity Reports, 4, 363-370. doi: 10.1007/ s13679-015-0169-4

World Health Organization (2000). Obesity: preventing and managing the global epidemic. (WHO Technical Report Series, No. 894). Recuperado de https://goo.gl/3WF5Tx

World Health Organization (2002). Diet, physical activity and health. Recuperado de http://apps.who.int/gb/ archive/pdf_files/WHA55/ea5516.pdf

World Health Organization (2004). Globalstrategy on diet, physical activity and health. Recuperado de https://goo.gl/ wYGwZG

World Health Organization (2008). School policy framework: Implementation of the WHO global strategy on diet, physical activity and health. Recuperado de https://goo.gl/cLNJ2C

\section{BY-NC-ND}

